\title{
Bariatric surgery and gastroesophageal reflux disease
}

\author{
Darius Ashrafi ${ }^{1}$, Emma Osland ${ }^{2,3}$, Muhammed Ashraf Memon ${ }^{1,4,5,6,7}$ \\ ${ }^{1}$ Mayne Medical School, School of Medicine, University of Queensland, Brisbane, Queensland, Australia; ${ }^{2}$ Department of Nutrition and Dietetics, \\ Royal Brisbane and Women's Hospital, Herston, Queensland, Australia; ${ }^{3}$ Department of Human Movements and Nutrition, University of \\ Queensland, Brisbane, Queensland, Australia; ${ }^{4}$ School of Agricultural, Computational and Environmental Sciences, International Centre for Applied \\ Climate Sciences and Centre for Health Sciences Research, University of Southern Queensland, Toowoomba, Queensland, Australia; ${ }^{5}$ Sunnybank \\ Obesity Centre South \& East Queensland Surgery (SEQS), Sunnybank, Queensland, Australia; ${ }^{6}$ Faculty of Health Sciences and Medicine, Bond \\ University, Gold Coast, Queensland, Australia; ${ }^{7}$ Faculty of Health and Social Science, Bolton University, Bolton, Lancashire, UK \\ Contributions: (I) Conception and design: MA Memon; (II) Administrative support: None; (III) Provision of study materials or patients: None; (IV) \\ Collection and assembly of data: All authors; (V) Data analysis and interpretation: All authors; (VI) Manuscript writing: All authors; (VII) Final \\ approval of manuscript: All authors. \\ Correspondence to: Professor Muhammed Ashraf Memon, FACS, FRACS, FRCS, Sunnybank Obesity Centre, Suite 9, McCullough Centre, 259 \\ McCullough Street, Sunnybank, QLD 4109, Australia. Email: mmemon@yahoo.com.
}

\begin{abstract}
With the rapidly increasing prevalence of obesity globally, the practice of bariatric surgery is being adopted routinely to prevent the development of chronic conditions as well as some forms of cancers associated with obesity. Gastroesophageal reflux disease (GERD) is one of those chronic conditions. Furthermore, there is accumulating data that obesity is associated with complications related to longstanding GERD such as erosive esophagitis (EE), Barrett's esophagus (BE), and esophageal adenocarcinoma (EAC). Central obesity, rather than body mass index (BMI), appears to be more closely associated with these complications. It should be expected, therefore, that weight loss procedures should result in improvement in GERD symptoms and its associated complications. However, in reality the different bariatric surgical procedures have unpredictable effects on an established GERD and may even produce GERD symptoms for the very first time (de novo). In this review, we explore the literature studying the effects of bariatric surgical operations on GERD. Roux-en-Y gastric bypass appears to have the most beneficial effect on GERD. On the other hand, laparoscopic sleeve gastrectomy and laparoscopic adjustable gastric banding (LAGB) are linked with long-term increased prevalence of GERD. We argue that GERD is an extremely important preoperative consideration for any patient undergoing bariatric surgery and therefore should be thoroughly investigated objectively (with 24-hour pH study and high-resolution manometry) to select the most suitable bariatric procedure for patients for their long-term success.
\end{abstract}

Keywords: Bariatric surgery; gastroesophageal reflux disease (GERD); laparoscopic adjustable gastric banding (LAGB); laparoscopic Roux-en-Y gastric bypass (LRYGB); laparoscopic sleeve gastrectomy

Submitted Aug 17, 2019. Accepted for publication Aug 30, 2019.

doi: $10.21037 /$ atm.2019.09.15

View this article at: http://dx.doi.org/10.21037/atm.2019.09.15

\section{Background}

Obesity is a global epidemic with increasing prevalence. The latest Centers for Disease Control and Prevention data shows that the prevalence in the United States has risen to $40 \%$ of adults and $18.5 \%$ in the young (1). Globally, an estimated 200 million men and 500 million women are obese, representing $10 \%$ of the world's population (2).

Obesity is a well-documented risk factor for many health conditions including cardiovascular disease, type-2 diabetes mellitus, osteoarthritis and some cancers (3). Furthermore, obesity is an important and independent risk factor for gastroesophageal reflux disease (GERD) and weight loss seems to be associated with significant improvement in 
symptoms of GERD (3).

The pathophysiology of GERD in adults is not fully understood but it is considered to be multifactorial (4). It is due to a mixture of hereditary and functional factors that result in abnormal relaxation of the lower esophageal sphincter (LES) or increased pressure from the stomach [e.g., from a hiatus hernia $(\mathrm{HH})$ or increased intraabdominal pressure] (5). The diagnosis of GERD is made clinically, with typical symptoms of heartburn, acid or water brash, regurgitation, dysphagia and odynophagia (6). However, atypical symptoms or extra-esophageal symptoms such as cough, sinusitis and pharyngitis can predominate and confuse the issue (6). The severity can only be assessed objectively by 24 or 48 hours ambulatory $\mathrm{pH}$ study or by a wireless $\mathrm{pH}$ Bravo monitoring device which can be utilized for up to 96 hours (7). Untreated GERD can result in reflux erosive esophagitis (EE), necrosis, ulceration, strictures and Barrett's esophagus (BE) (8). Ten percent of cases of BE can progress to esophageal adenocarcinoma (EAC) (6).

What is the relationship between obesity and GERD? Abdominal obesity has been postulated to promote GERD by elevating intra-abdominal pressure, which promotes reflux and the development of $\mathrm{HH}$ (9). Studies comparing computerized tomography-measured abdominal fat composition showed that patients with $\mathrm{EE}, \mathrm{BE}$ and $\mathrm{EAC}$ had greater intra-abdominal visceral adiposity than controls (10). The metabolically active visceral abdominal fat may also predispose to BE and EAC by GERD-independent mechanisms that promote the progression from inflammation to metaplasia and neoplasia; these include alterations in the levels of adipokines, cytokines and chemokines, as well as the insulin/insulin growth factor pathways (11). Hampel et al. conducted a meta-analysis exploring the link between obesity and GERD (12). Nine studies were included and six found a statistically significant association with obesity and GERD. Increasing body mass index (BMI) was also associated with progressive risk of GERD, EE and EAC, likely driven by increased intra-abdominal pressure and the gastroesophageal pressure gradient.

Bariatric surgery has demonstrated significant and durable weight loss. It has significantly evolved over the last decade. The current bariatric surgery procedures offered include restrictive procedures such as the laparoscopic vertical sleeve gastrectomy (LVSG) and laparoscopic adjustable gastric banding (LAGB) or malabsorptive procedures such as laparoscopic Roux-en-Y gastric bypass (LRYGB) (3). The case-volumes in the United States have shifted with most recent data showing LVSG accounting for $60 \%$ of bariatric surgery while $18 \%$ are LRYGB and $3 \%$ are LAGB (13). In theory, bariatric surgery should result in reduced GERD through facilitating sustainable weight loss. However, studies have demonstrated varying responses of GERD to bariatric surgery, depending on which surgery is performed. Therefore, the preoperative evaluation of all such patients is important in preparation for bariatric surgery as no one operation is appropriate for all patients. In 2008, the American Society for Gastrointestinal Endoscopy recommended a preoperative upper gastrointestinal endoscopy for all bariatric surgery patients due to the high number of incidental clinically significant findings which include $\mathrm{HH}$, esophagitis, gastritis, $\mathrm{BE}$, peptic ulcer disease or upper gastrointestinal tumors (14). Barium swallow studies can be an important adjunct to identify $\mathrm{HH}$, however, they are notoriously underestimate the presence of $\mathrm{HH}$ if static images are obtained and therefore dynamic fluoroscopic study should always be performed. In more recent guidelines, the recommendations are more lenient with the decision to perform preoperative upper gastrointestinal endoscopy to be individualized and tailored to the surgeon, the patient and the type of bariatric surgery (15). The potential benefits of undertaking these preoperative assessments remains valid.

The aim of the present review article was to assess the impact of various bariatric surgical procedures, not on weight loss, but GERD.

\section{LVSG and GERD}

LVSG is popular due to its relative simplicity compared to other bariatric operations and success in providing sustained weight loss. However, studies describing its effects on GERD have demonstrated conflicting outcomes, and there is growing evidence that it may have negative impact on GERD.

Stenard and Iannelli conducted the largest systematic review of LVSG and GERD which included 25 studies (16). The findings were mixed. Thirteen studies found worsening of GERD post-LVSG across 5,953 patients with a mean BMI of $42 \pm 4 \mathrm{~kg} / \mathrm{m}^{2}$ (range, $37-55.5 \mathrm{~kg} / \mathrm{m}^{2}$ ) and mean follow up of 29 \pm 22 months (range, 3-72 months) (16). Only one study was prospective whereas the rest were retrospective. The evaluation of GERD was heterogenous, and although all studies had preoperative endoscopy data, postoperative evaluation varied (16). These included esophageal manometry, contrast studies and 24-hour ambulatory $\mathrm{pH}$ studies, with the majority including subjective clinical evaluation by means of symptoms of validated questions (e.g., Montreal's criteria) (16). Twelve studies found clinical 
improvement on GERD across 1,863 patients, with a mean BMI of $51 \pm 13 \mathrm{~kg} / \mathrm{m}^{2}$ (range, $36.5-65 \mathrm{~kg} / \mathrm{m}^{2}$ ) and mean

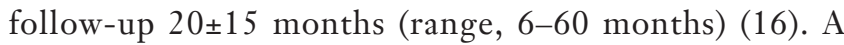
majority of the studies were based on clinical evaluation, again with only a few utilizing endoscopy, 24-hour ambulatory $\mathrm{pH}$ studies, esophageal manometry or contrast studies (16). With the aforementioned findings, the authors proposed caution with performing LVSG due to the unquantified potential for worsening rates of GERD (16).

Chiu et al. conducted a systemic review of 15 studies analyzing the effects of LVSG on GERD (17). Again, there was discordance with four studies showing an increase in GERD after LVSG while seven studies showing an improvement (17). Three studies only included the postoperative prevalence of GERD and one did not include any data on both pre- and postoperative prevalence (17). For the studies where overall prevalence of GERD following LVSG reduced, it was noted that patients with pre-existing GERD had shown improvement, however, new GERD developed in a proportion of patients (17). However, Chiu et al. did not quantify the number or proportion of new cases of GERD and therefore it is difficult to make any firm conclusions based on retrospective nature of this review and subjective provision of the data (17).

Oor et al. conducted the first meta-analysis on this topic and like the authors above, they were unable to provide uniformed consensus conclusions due to the high heterogeneity of available studies (18). They included 33 studies with 8,092 patients undergoing LVSG (18). The majority of the studies $(\mathrm{k}=30)$ reported clinical evaluation of GERD symptoms postoperatively, while four included use of anti-acid medications and eight studies utilized functional tests (18). Interestingly, the studies utilizing validated questionnaires had a risk difference of $4.3 \%$ postoperatively, while the eight studies with functional tests had paradoxical results (18). The pooled incidence of de novo GERD was $20 \%$. Given this, the authors made no definite conclusions, stating only that there appears to be an increased prevalence of GERD symptoms post LVSG (18).

Himpens et al. conducted a randomized study with one and three year follow-up comparing LAGB and LVSG (19). Eighty patients were randomized equally into each arm. GERD was determined by the usage and change of usage of proton pump inhibitors (PPIs) (19). They found that $75 \%$ of patients who underwent LVSG with pre-existing GERD had resolution, however, $21.8 \%$ new cases developed at the first year (19). By three years, only 3.1\% reported GERD (19).

The anatomical and physiological effects on GERD post LVSG have multi-factorial explanations. In cases of de novo GERD, it is thought to be due to decreased gastric emptying, reduced LES pressure, blunting of the angle of His, decreased compliance and reduced volume of the stomach and increased intra-gastric pressure secondary to the narrow gastric pouch and herniation of part of the sleeve into the mediastinum $(17,20)$. Indeed, several investigators such as Braghetto et al. have shown patients with LVSG to have reduced LES pressure via esophageal manometry (21). Himpens et al. postulated that a lack of gastric compliance, emptying and blunting of angle of His was responsible for GERD at one year, and that this compliance improved by three years accounting for the reduced de novo GERD (19).

The shape of the sleeve itself can be a significant cause of GERD. Keidar et al. studied 716 patients who underwent a LVSG and suffered GERD postoperatively (22). Using barium swallow studies, they identified that all 716 patients had a dilated upper sleeve with a relative narrowing of the mid-stomach (22).

Genco et al. evaluated the incidence of GERD in patients undergoing LVSG with pre- and postoperative endoscopic and histologic examinations (23). Of 110 patients, they found a significant increase in GERD symptoms and PPI intake (23). They also found a significant increase in the incidence of EE, as well as new diagnosis of $\mathrm{BE}$ in $17.2 \%$ of patients. GERD is, therefore, an important long-term complication to consider and evaluate post-LVSG (23). Genco et al. advocated for endoscopic surveillance after LVSG, irrespective of the presence of GERD symptoms (23).

In view of these results, the improvement in GERD postLVSG can be postulated to be anatomical (e.g., resection of acid-producing gastric fundus, accelerated gastric emptying and reduced gastric volume) or systemic (reduced intraabdominal pressure due to weight loss).

The role of $\mathrm{HH}$ repair in bariatric surgery is an important consideration for patients with GERD. Concurrent LVSG with $\mathrm{HH}$ repair has been explored with more contemporary studies showing supportive evidence of improvement in GERD. Soricelli et al. studied 378 bariatric patients undergoing LVSG (24). Sixty patients had pre-operative GERD and 42 patients had a pre-operative diagnosis of $\mathrm{HH}$ (24). Intra-operatively, a further 55 patients were found to have HH (24). Ninety-seven patients underwent concurrent LSVG with HH repair (24). They found significantly higher rates of de novo GERD symptoms in patients who did not undergo a concurrent $\mathrm{HH}$ repair with their LVSG $(22.9 \%$ vs. $0 \%, \mathrm{P}=0.01$ ) (24). Daes et al. also found resolution of GERD in $94 \%$ of their patients (25). In their study cohort of 
134 patients, 34 were found to have $\mathrm{HH}$ (25). Twenty-nine of these patients had preoperative GERD (25). All 34 underwent LVSG with HH repair and at 6 to 12 months follow up only 2 of 134 patients had symptoms of GERD (25). Both patients had preoperative large $\mathrm{HH}$ with a persistent small $\mathrm{HH}$ diagnosed on post-operative endoscopy (25).

LVSG should be carefully considered for patients with significant preoperative GERD. They may be suitable for alternative bariatric operations. Similarly, there should be an awareness for the potential development or worsening of GERD.

\section{LAGB and GERD}

LAGB has had mixed results with GERD. de Jong et al. conducted a systemic review to explore this (26). There were 20 studies with 3,307 patients (26). There was a significant reduction in GERD symptoms reported from $33.7 \%$ preoperatively to $7.7 \%$ postoperatively, and PPI usage reduced from $27.5 \%$ to $9.5 \%$ (26). A subset of patients developed de novo GERD (15\%) and new onset of reflux esophagitis (29.4\%) (26).

Chen et al. explored this further (27). They performed highresolution esophageal manometry and 24-hour ambulatory esophageal $\mathrm{pH}$ study on three groups: asymptomatic LAGB $(\mathrm{n}=14)$, symptomatic LAGB $(\mathrm{n}=20)$ and preoperative reflux patients $(n=20)(27)$. They found that symptomatic patients had a significantly larger gastric pouch with higher esophageal acid exposure $(\mathrm{P}<0.001)(27)$. The symptomatic group also had significantly LES resting tone and impaired contractility of the lower esophageal segment (27).

Himpens et al. found that although GERD was only present in $8.8 \%$ of patients at one year, by three years $20.5 \%$ of LAGB patients had developed de novo GERD, following the opposite trend to LVSG (19). It appears that LAGB provides short-term improvement of GERD symptoms (19). The subset of patients who develop new onset disease, it is difficult to tell whether this is from pathologic GERD or complications of LAGB (19). It is postulated that the band placement may create a highpressure system promoting GERD (19). The authors did not explore or discuss the effects of volume of filling within the band for LAGB patients or whether emptying the band improved symptoms (19).

In summary, there is evidence to support the shortterm improvement with GERD after LAGB. However, there appears to be a proportion of patients who develop de novo GERD or following gastric band complication such as slippage of band in the long-term.

\section{LRYGB and GERD}

LRYGB is considered most effective bariatric procedure for GERD $(3,28)$. Indeed, it has been recommended as the procedure of choice for patients with intractable GERD following standard anti-reflux surgery, although, this notion is increasingly being challenged in recent literature.

In patients with severe GERD symptoms post LVSG, with resistance to maximal medical therapy, revisional surgery has been advocated: LRYGB is the procedure of choice in these patients. Cheung et al. conducted a systematic review of studies examining patients undergoing revisional bariatric surgery for failed weight loss (29). GERD was assessed in three studies, and found that all patients $(n=15)$ had complete resolution of GERD symptoms with repeat LRYGB (29). Parmar et al. converted 22 LVSG to LRYGB and found that $100 \%$ of patients reported improvement in GERD symptoms, and $80 \%$ were able to cease medications (30).

Many studies have shown significant improvement in GERD with LRYGB. Frezza et al. studied 152 patients with pre-existing GERD who underwent LRYGB (31). There was a significant decrease in all GERD-related symptoms including heartburn, water brash, wheezing, laryngitis and aspiration $(\mathrm{P}<0.01)$. The use of medications dropped significantly for both PPI and $\mathrm{H}_{2}$ blockers $(\mathrm{P}<0.01)(31)$. Perry et al. performed LRYGB on 57 patients with persistent GERD. At a mean follow-up of 18 months, all patients experienced a clinical improvement or no symptoms of GERD (32).

Madalosso et al. studied 53 patients and evaluated them for GERD preoperatively, at six months and at 39 months post-surgery (33). There was a statistically significant reduction in GERD as assessed by the Montreal Consensus as well as an esophageal acid exposure assessment (33). They found the reduced incidence of GERD and reflux esophagitis was statistically significantly between preoperative, six and 39 months (33).

Mendes-Filho et al. conducted a systematic review exploring conversion of previous anti-reflux surgeries to LRYGB for refractory GERD (34). They found six studies where gastric bypass was used, with a total of 121 patients (34). Ninety-nine of the patients underwent LRYGB while 22 underwent open RYGB (34). One hundred and six patients had total remission of symptoms, while 12 showed partial improvement (34). In the studies which included use of PPI, 29 out of 35 patients ceased their use (34).

Recently, Holmberg et al. has contested this long-standing 
notion and postulated that the effect has been overstated (35). They studied all adult patients who underwent RYGB in Sweden which included 2,454 patients (35). The median follow-up was 4.6 years (IQR 3.1-6.3). They defined postoperative reflux as residual or recurring symptoms of GERD with use of acid suppression medications for beyond six months postoperatively (35). Interestingly, they found GERD persisted in $48.8 \%$ of patients within two years of RYGB and persisted for up to 10 years after surgery (35). The strongest risk factor for postoperative GERD was highdose preoperative acid suppression (35). In comparison, less than $10 \%$ of patients have persisting GERD after the traditional anti-reflux surgery of fundoplication (35). Despite these findings, the authors concede that RYGB remains the most effect bariatric procedure in reducing GERD for the aforementioned reasons (35).

\section{Comparison studies}

High quality trials have been challenging in bariatric surgery. There have been very few randomized controlled trials (RCT); most have been small feasibility studies for larger trials that did not progress to recruitment. Existing prospective studies have been very heterogenous in patient selection, operative approach, definition, diagnosis and evaluation of GERD. The following are current landmark prospective trials in this area.

The Swiss Multicenter Bypass or Sleeve Study (SMBOSS) is an RCT comparing LRYGB and LVSG (36). Two hundred and seventeen patients were enrolled and randomly assigned to undergo LRYGB $(\mathrm{n}=110)$ or LVSG $(\mathrm{n}=107)(36)$. GERD remission was observed more frequently after LRYGB (60.4\%) than after LVSG (25.0\%) (36). GERD worsened (as defined by more symptoms or increase in acid suppressing therapy) more often after LVSG (31.8\%) than after LRYGB (6.3\%) (36). At five years, de novo GERD developed in $32 \%$ of LVSG patients compared to $11 \%$ of LRYGB patients (36). Nine percent of patients who underwent LVSG required a subsequent LRYGB to manage their refractory symptoms of GERD (36).

Similarly, but in a smaller RCT $(\mathrm{n}=100)$, Ignat et al. report two LVSG conversions to RYGB for severe GERD, with additional patients requiring medical management (37). However, the interpretation of these outcomes is confounded by the lack of description regarding the presence or absence of $\mathrm{HH}$ in patients receiving surgery. Similarly, concomitant $\mathrm{HH}$ repair within procedures and the preoperative use of physiological testing, such as 24-hour $\mathrm{pH}$ study and high- resolution manometry, is not consistently reported.

The Sleeve $v s$. Bypass (SLEEVEPASS) study was another RCT comparing long-term outcomes between LVSG and LRYGB (38). This was a multicentered, multi-surgeon randomized control trial in Finland. Two hundred and forty patients were enrolled and randomized to undergo LVSG $(n=121)$ or LRYGB $(n=119)$ (38). GERD was not a primary or secondary outcome, and all those with severe GERD preoperatively or with large $\mathrm{HH}$, were excluded from the study (38). This study also included five-year follow-up with $80.4 \%$ of patients reaching it (38). At five years, $6 \%$ of the LVSG patients required conversion from sleeve to LRYGB for severe reflux, while $9 \%$ required daily PPI. GERD was not reported in the LRYGB group (38).

Zhang et al. also conducted an RCT comparing longterm outcomes of LVSG and LRYGB. However, they did not explore GERD outcomes (39).

Biter et al. randomized 150 patients to LVSG or LRYGB, and compared their quality of life at preoperative, 2 and 12 months (40). They used the GerdQ (GERD Questionnaire) score only to assess reflux, with a higher score denoting more reflux (40). At 12 months there was an $85 \%$ completion rate (40). The GerdQ score increased significantly for patients undergoing LVSG at 2 and 12 months, while it decreased significantly for patients undergoing LRYGB (40).

BOLD (Bariatric Outcomes Longitudinal Database) is a prospective database of patients undergoing bariatric surgery. Using this database, Pallati et al. identified 36,938 patients who had preoperative evidence of GERD (41). After excluding patients with concomitant $\mathrm{HH}$ repair or fundoplication, there were 22,830 patients with six months follow up. Mean age was $47.6 \pm 11.1$ years, with an $82 \%$ female population. Mean BMI was $46.3 \pm 8.0 \mathrm{~kg} / \mathrm{m}^{2}$ (41). Patients undergoing LRYGB, LAGB and LVSG all demonstrated significant reduction in their GERD symptom score (6-point scale). The GERD score improvement was maximal in LRYGB patients, followed by LAGB and then LVSG (41).

\section{Conclusions}

Obesity is an important risk factor for GERD. While bariatric surgery is the most effective and sustainable treatment for obesity, GERD must be considered for all patients undergoing such procedures. LRYGB has shown superiority over LVSG for alleviation and management of GERD symptoms from the available literature. It has 
reported efficiency similar to Nissen fundoplication and is the options with refractory GERD post-fundoplication in obese patients. A patient's preoperative status should be evaluated to optimize their choice of bariatric surgery: patient selection is key. In appropriately worked-up patients who have GERD, LRYGB could be considered as the first option. In patients with $\mathrm{HH}$, this should be evaluated, and treatment should be considered during their bariatric surgery. While LVSG continues to become more commonly performed, it is important to monitor these patients for the long-term risks of developing GERD and its sequalae. In case of troublesome GERD symptoms following LVSG and LAGB, revisional surgery with LRYGB seems to be the best option.

\section{Acknowledgments}

Funding: None.

\section{Footnote}

Provenance and Peer Review: This article was commissioned by the Guest Editor (Muhammed Ashraf Memon) for the focused issue "Bariatric Surgery" published in Annals of Translational Medicine. The article was sent for external peer review organized by the Guest Editor and the editorial office.

Conflicts of Interest: The focused issue "Bariatric Surgery" was commissioned by the editorial office without any funding or sponsorship. MAM served as the unpaid Guest Editor of the focused issue. The other authors have no conflicts of interest to declare.

Ethical Statement: The authors are accountable for all aspects of the work in ensuring that questions related to the accuracy or integrity of any part of the work are appropriately investigated and resolved.

Open Access Statement: This is an Open Access article distributed in accordance with the Creative Commons Attribution-NonCommercial-NoDerivs 4.0 International License (CC BY-NC-ND 4.0), which permits the noncommercial replication and distribution of the article with the strict proviso that no changes or edits are made and the original work is properly cited (including links to both the formal publication through the relevant DOI and the license). See: https://creativecommons.org/licenses/by-nc-nd/4.0/.

\section{References}

1. Hales CM, Carroll MD, Fryar CD, et al. Prevalence of Obesity Among Adults and Youth: United States, 2015-2016. NCHS data brief, No. 288. Hyattsville, MD: National Center for Health Statistics. 2017.

2. Lean MEJ, Astrup A, Roberts SB. Making progress on the global crisis of obesity and weight management. BMJ 2018;361:k2538.

3. El-Hadi M, Birch DW, Gill RS, et al. The effect of bariatric surgery on gastroesophageal reflux disease. Can J Surg 2014;57:139-44.

4. Boeckxstaens G, El-Serag HB, Smout AJ, et al. Symptomatic reflux disease: the present, the past and the future. Gut 2014;63:1185-93.

5. Vakil N, van Zanten SV, Kahrilas P, et al. The Montreal definition and classification of gastroesophageal reflux disease: a global evidence-based consensus. Am J Gastroenterol 2006;101:1900-20; quiz 43.

6. Bennett C, Vakil N, Bergman J, et al. Consensus statements for management of Barrett's dysplasia and early-stage esophageal adenocarcinoma, based on a Delphi process. Gastroenterology 2012;143:336-46.

7. Kessels SJM, Newton SS, Morona JK, et al. Safety and Efficacy of Wireless $\mathrm{pH}$ Monitoring in Patients Suspected of Gastroesophageal Reflux Disease: A Systematic Review. J Clin Gastroenterol 2017;51:777-88.

8. Chiejina M, Samant H. Esophageal Ulcer. StatPearls. Treasure Island (FL), 2019.

9. Boeckxstaens G, El-Serag HB, Smout AJ, et al. Republished: symptomatic reflux disease: the present, the past and the future. Postgrad Med J 2015;91:46-54.

10. El-Serag HB, Hashmi A, Garcia J, et al. Visceral abdominal obesity measured by CT scan is associated with an increased risk of Barrett's oesophagus: a case-control study. Gut 2014;63:220-9.

11. Duggan C, Onstad L, Hardikar S, et al. Association between markers of obesity and progression from Barrett's esophagus to esophageal adenocarcinoma. Clin Gastroenterol Hepatol 2013;11:934-43.

12. Hampel H, Abraham NS, El-Serag HB. Meta-analysis: obesity and the risk for gastroesophageal reflux disease and its complications. Ann Intern Med 2005;143:199-211.

13. Estimate of Bariatric Surgery Numbers, 2011-2017. American Society for Metabolic and Bariatric Surgery. 2018. Available online: https://asmbs.org/resources/ estimate-of-bariatric-surgery-numbers

14. Greenwald D. Preoperative gastrointestinal assessment before bariatric surgery. Gastroenterol Clin North Am 2010;39:81-6.

15. ASGE Standards of Practice Committee, Anderson MA, Gan SI, et al. Role of endoscopy in the bariatric surgery 
patient. Gastrointest Endosc 2008;68:1-10.

16. Stenard F, Iannelli A. Laparoscopic sleeve gastrectomy and gastroesophageal reflux. World J Gastroenterol 2015;21:10348-57.

17. Chiu S, Birch DW, Shi X, et al. Effect of sleeve gastrectomy on gastroesophageal reflux disease: a systematic review. Surg Obes Relat Dis 2011;7:510-5.

18. Oor JE, Roks DJ, Unlu C, et al. Laparoscopic sleeve gastrectomy and gastroesophageal reflux disease: a systematic review and meta-analysis. Am J Surg 2016;211:250-67.

19. Himpens J, Dapri G, Cadiere GB. A prospective randomized study between laparoscopic gastric banding and laparoscopic isolated sleeve gastrectomy: results after 1 and 3 years. Obes Surg 2006;16:1450-6.

20. Patti MG, Schlottmann F. Gastroesophageal Reflux After Sleeve Gastrectomy. JAMA Surg 2018;153:1147-8.

21. Braghetto I, Lanzarini E, Korn O, et al. Manometric changes of the lower esophageal sphincter after sleeve gastrectomy in obese patients. Obes Surg 2010;20:357-62.

22. Keidar A, Appelbaum L, Schweiger C, et al. Dilated upper sleeve can be associated with severe postoperative gastroesophageal dysmotility and reflux. Obes Surg 2010;20:140-7.

23. Genco A, Soricelli E, Casella G, et al. Gastroesophageal reflux disease and Barrett's esophagus after laparoscopic sleeve gastrectomy: a possible, underestimated long-term complication. Surg Obes Relat Dis 2017;13:568-74.

24. Soricelli E, Iossa A, Casella G, et al. Sleeve gastrectomy and crural repair in obese patients with gastroesophageal reflux disease and/or hiatal hernia. Surg Obes Relat Dis 2013;9:356-61.

25. Daes J, Jimenez ME, Said N, et al. Laparoscopic sleeve gastrectomy: symptoms of gastroesophageal reflux can be reduced by changes in surgical technique. Obes Surg 2012;22:1874-9.

26. de Jong JR, Besselink MG, van Ramshorst B, et al. Effects of adjustable gastric banding on gastroesophageal reflux and esophageal motility: a systematic review. Obes Rev 2010;11:297-305.

27. Chen RY, Burton PR, Ooi GJ, et al. The Physiology and Pathophysiology of Gastroesophageal Reflux in Patients with Laparoscopic Adjustable Gastric Band. Obes Surg 2017;27:2434-43.

28. Angrisani L, Santonicola A, Iovino P, et al. Bariatric Surgery Worldwide 2013. Obes Surg 2015;25:1822-32.

29. Cheung D, Switzer NJ, Gill RS, et al. Revisional bariatric surgery following failed primary laparoscopic sleeve gastrectomy: a systematic review. Obes Surg 2014;24:1757-63.

30. Parmar CD, Mahawar KK, Boyle M, et al. Conversion of Sleeve Gastrectomy to Roux-en-Y Gastric Bypass is
Effective for Gastro-Oesophageal Reflux Disease but not for Further Weight Loss. Obes Surg 2017;27:1651-8.

31. Frezza EE, Ikramuddin S, Gourash W, et al. Symptomatic improvement in gastroesophageal reflux disease (GERD) following laparoscopic Roux-en-Y gastric bypass. Surg Endosc 2002;16:1027-31.

32. Perry Y, Courcoulas AP, Fernando HC, et al. Laparoscopic Roux-en-Y gastric bypass for recalcitrant gastroesophageal reflux disease in morbidly obese patients. JSLS 2004;8:19-23.

33. Madalosso CA, Gurski RR, Callegari-Jacques SM, et al. The Impact of Gastric Bypass on Gastroesophageal Reflux Disease in Morbidly Obese Patients. Ann Surg 2016;263:110-6.

34. Mendes-Filho AM, Godoy ES, Alhinho H, et al. Fundoplication Conversion in Roux-En-Y Gastric Bypass for Control of Obesity and Gastroesophageal Reflux: Systematic Review. Arq Bras Cir Dig 2017;30:279-82.

35. Holmberg D, Santoni G, Xie S, et al. Gastric bypass surgery in the treatment of gastro-oesophageal reflux symptoms. Aliment Pharmacol Ther 2019;50:159-66.

36. Peterli R, Wolnerhanssen BK, Peters T, et al. Effect of Laparoscopic Sleeve Gastrectomy vs Laparoscopic Rouxen-Y Gastric Bypass on Weight Loss in Patients With Morbid Obesity: The SM-BOSS Randomized Clinical Trial. JAMA 2018;319:255-65.

37. Ignat M, Vix M, Imad I, et al. Randomized trial of Rouxen-Y gastric bypass versus sleeve gastrectomy in achieving excess weight loss. Br J Surg 2017;104:248-56.

38. Salminen P, Helmio M, Ovaska J, et al. Effect of Laparoscopic Sleeve Gastrectomy vs Laparoscopic Rouxen-Y Gastric Bypass on Weight Loss at 5 Years Among Patients With Morbid Obesity: The SLEEVEPASS Randomized Clinical Trial. JAMA 2018;319:241-54.

39. Zhang Y, Zhao H, Cao Z, et al. A randomized clinical trial of laparoscopic Roux-en-Y gastric bypass and sleeve gastrectomy for the treatment of morbid obesity in China: a 5-year outcome. Obes Surg 2014;24:1617-24.

40. Biter LU, van Buuren MMA, Mannaerts GHH, et al. Quality of Life 1 Year After Laparoscopic Sleeve Gastrectomy Versus Laparoscopic Roux-en-Y Gastric Bypass: a Randomized Controlled Trial Focusing on Gastroesophageal Reflux Disease. Obes Surg 2017;27:2557-65.

41. Pallati PK, Shaligram A, Shostrom VK, et al. Improvement in gastroesophageal reflux disease symptoms after various bariatric procedures: review of the Bariatric Outcomes Longitudinal Database. Surg Obes Relat Dis 2014;10:502-7.

Cite this article as: Ashrafi D, Osland E, Memon MA. Bariatric surgery and gastroesophageal reflux disease. Ann Transl Med 2020;8(Suppl 1):S11. doi: 10.21037/atm.2019.09.15 